\title{
REGULAR BOUNDARY ELEMENTS
}

\author{
ROBIN HARTE
}

ABSTRACT. In a Banach algebra the regular elements in the closure of the group of invertibles are in a generalized sense "of index zero".

1. If $A$ is a ring, with identity 1 and invertible group $A^{-1}$, or more generally [6] an additive category, we shall write

$$
\overparen{A}=\{a \in A: a \in a A a\}
$$

for the regular or "relatively Fredholm" elements of $A$, and

$$
A^{-1} \dot{A}=\dot{A} A^{-1}=\left\{a \in A: a \in a A^{-1} a\right\}
$$

for the decomposably regular or "relatively Weyl" elements of $A$; these include the invertible elements $A^{-1}$, and also the idempotents

$$
\dot{A}=\left\{a \in A: a^{2}=a\right\} \text {. }
$$

1.1 THEOREM. If $A$ is a Banach algebra then

$$
A^{-1} \dot{A}=\overparen{A} \cap \operatorname{cl}\left(A^{-1}\right) \text {. }
$$

Proof. The decomposably regular elements are always regular; if $A$ is a normed algebra and if $a \in A$ is decomposably regular then we can write

$$
a=c p=q c \text { with } c \in A^{-1}, p \in \dot{A}, q \in \dot{A} ;
$$

we have anticipated this in the notation for (1.0.2). Putting for each $n$

$$
b_{n}=c(p+(1 / n)(1-p)), \quad b_{n}^{\prime}=(p+n(1-p)) c^{-1}
$$

gives

$$
\left\|a-b_{n}\right\| \rightarrow 0 \text { and } b_{n}^{\prime} b_{n}=1=b_{n} b_{n}^{\prime},
$$

so that also $a \in \operatorname{cl}\left(A^{-1}\right)$. Conversely, without restriction on $A$, we claim that it is sufficient, for $a \in A$ to be decomposably regular, that there are $a^{\prime} \in A$ and $b \in A$ for which

(1.1.5) $a=a a^{\prime} a$ and $a^{\prime}=a^{\prime} a a^{\prime}$ and $b \in A^{-1}$ and $1+(b-a) a^{\prime} \in A^{-1}$.

Indeed if (1.1.5) holds and we define

$$
a^{\prime \prime}=a^{\prime}+\left(1-a^{\prime} a\right) b^{-1}\left(1-a a^{\prime}\right)
$$

Received by the editors December 4, 1985 .

1980 Mathematics Subject Classification. Primary 47B30, 46B30; Secondary 47A10, 47A53.

Key words and phrases. Regular, generalized inverse, Fredholm, Fredholm of index zero, boundary. 
then $a^{\prime \prime}$ is an invertible generalised inverse for $a$. To see this write

$$
p=a^{\prime} a, \quad q=a a^{\prime},
$$

$$
k^{\prime}=\left(1+(b-a) a^{\prime}\right)^{-1}, \quad k^{\prime \prime}=1-a^{\prime} k^{\prime}(b-a)=\left(1+a^{\prime}(b-a)\right)^{-1} ;
$$

then

$a=a a^{\prime \prime} a$ and $\left(a+(1-q) k^{\prime} b(1-p)\right) a^{\prime \prime}=1=a^{\prime \prime}\left(a+(1-q) b k^{\prime \prime}(1-p)\right)$, since

(1.1.9) $k^{\prime} b p=a=q b k^{\prime \prime} \quad$ and $\quad k^{\prime}(1-q)=1-q \quad$ and $\quad(1-p) k^{\prime \prime}=1-p$.

The argument that (1.1.5) and (1.1.6) imply (1.1.8) works in an arbitrary ring or an additive category; if we specialise to a Banach algebra then we can ensure the last part of (1.1.5) by asking that

$$
\|b-a\|\left\|a^{\prime}\right\|<1 \text {. }
$$

2. The extreme simplicity of the statement and proof of Theorem 1.1 conceals a long gestation and a difficult birth. In 1977 Treese and Kelly [8] claimed a slightly stronger result for the algebra $A$ of operators on a Banach space $X$. Unfortunately their argument fails; recently Gonzalez [3] has found a counterexample. Gonzalez also gives a proof of Theorem 1.1 for operators; his argument rests on a theorem of Caradus [1, Chapter 5, Theorem 13] involving two kinds of "gap" between subspaces. Part of the proof of Caradus' theorem is taken from Kato [7, Chapter IV]; unfortunately there is a subtle difference between the definitions given by Caradus and by Kato. The argument we have given for Theorem 1.1 was, however, discovered by following the trail marked out by Gonzalez [3]. It is clear that the result extends to those possibly incomplete algebras [2] for which the invertible group is open.

When $A$ is the algebra of operators on a Banach space and $a \in A$ is a Fredholm operator then the conclusion of Theorem 1.1 follows from the continuity of the index. More generally, if $T: A \rightarrow B$ is a homomorphism of rings, or an additive functor between categories, there is inclusion $[5,6]$.

$$
A^{-1} \subseteq A^{-1}+T^{-1}(0) \subseteq T^{-1}\left(B^{-1}\right)
$$

We shall call $T^{-1}\left(B^{-1}\right)$ the $T$-Fredholm elements of $A$ and $A^{-1}+T^{-1}(0)$ the $T$-Weyl elements. If $A$ is the algebra of bounded operators and $T$ takes the quotient by the ideal of compact operators then these are, respectively, the Fredholm operators and the Fredholm operators of index zero; if instead $A$ is the continuous functions on the disc and $T$ restricts to the circle then the "Fredholm" functions do not vanish on the circle, and for the "Weyl" functions the "winding number" or topological degree of the boundary value function is zero. The continuity of the index in each case predicts that the "Weyl" elements should be open and closed in the "Fredholm" elements.

2.1 THEOREM. If $T: A \rightarrow B$ is a continuous homomorphism of Banach algebras which is decomposably regular in the sense that

$$
T^{-1}\left(B^{-1}\right) \subseteq \overparen{A} \text { and } 1+T^{-1}(0) \subseteq A^{-1} \dot{A}
$$


then $A^{-1}+T^{-1}(0)$ is open and closed in $T^{-1}\left(B^{-1}\right)$ :

$$
A^{-1}+T^{-1}(0)=\operatorname{int}\left(A^{-1}+T^{-1}(0)\right)=T^{-1}\left(B^{-1}\right) \cap \operatorname{cl}\left(A^{-1}\right) .
$$

Proof. If $A^{-1}$ is open in $A$ then so is $A^{-1}+T^{-1}(0)$. More generally, in an additive category $A$ we have

$$
1-a^{\prime} a \in T^{-1}(0), \quad a^{\prime} \in A^{-1}, \quad 1+a^{\prime}(b-a) \in A^{-1} \Rightarrow b \in A^{-1}+T^{-1}(0) .
$$

For the second part of (2.1.2) we combine (1.1.1) with

$$
A^{-1}+T^{-1}(0)=T^{-1}\left(B^{-1}\right) \cap A^{-1} \stackrel{\circ}{ }
$$

which is a consequence of the decomposable regularity (2.1.1). Forward inclusion in (2.1.4) follows at once from (2.1.1); conversely if $a=a a^{\prime} a$ with $a^{\prime}$ invertible in $A$ and $T(a)$ invertible in $B$ then

$$
1-a^{\prime} a \in T^{-1}(0) \text { and } a=\left(a^{\prime}\right)^{-1}+\left(a^{\prime}\right)^{-1}\left(1-a^{\prime} a\right) .
$$

The decomposable regularity condition (2.1.1) fails if $T: A \rightarrow B$ is restriction to the circle for continuous functions on the disc. In this case (2.1.2) still holds, but for a different reason: $T$ is onto and the invertible group $A^{-1}$ is connected. If we write [4]

$$
\operatorname{Exp}(A)=\left\{e^{c_{1}} e^{c_{2}} \cdots e^{c_{k}}: k \in \mathbb{N}, c \in A^{k}\right\}
$$

for the connected component of the identity in the topological group $A^{-1}$, then we are assuming

$$
T(A)=B \text { and } A^{-1}=\operatorname{Exp}(A) .
$$

But now [4, Theorem 2]

$$
A^{-1}+T^{-1}(0)=\operatorname{Exp}(A)+T^{-1}(0)=T^{-1}(\operatorname{Exp}(B)),
$$

and the right-hand side is both open and closed in $T^{-1}\left(B^{-1}\right)$.

\section{REFERENCES}

1. S. R. Caradus, Generalized inverses and operator theory, Queen's Papers in Pure and Appl. Math., no. 50, Queen's University, Kingston, Ontario, 1978.

2. R. Fuster and A. Marquina, Geometric series in incomplete normed algebras, Amer. Math. Monthly 91 (1984), 49-51.

3. M. Gonzalez, A perturbation result for generalized Fredholm operators in the boundary of the group of invertible operators, Proc. Roy. Irish Acad. Sect. A 86 (1987).

4. R. E. Harte, The exponential spectrum in Banach algebras, Proc. Amer. Math. Soc. 56 (1976), 114-118.

5. ___ Fredholm theory relative to a Banach algebra homomorphism, Math. Z. 179 (1982), 431-436.

6. —_ Fredholm, Weyl and Browder theory, Proc. Roy. Irish Acad. Sect. A 85 (1986), 151-176.

7. T. Kato, Perturbation theory for linear operators, Springer-Verlag, Berlin and New York, 1966.

8. G. W. Treese and E. P. Kelly, Generalized Fredholm operators and the boundary of the maximal group of invertible operators, Proc. Amer. Math. Soc. 67 (1977), 123-128.

Department of Mathematics, University College, Cork, Ireland

Department of Mathematics, University of Iowa, Iowa City, Iowa 52242 